Nol ar absor pt i on coef $\mathrm{fi}$ ci ent and radi ol yt i c yi el d of solvated el ectrons in

di et hyl met hyl ( 2- met hoxy) ammoni um bi s ( tri fl uor omet hanesul fonyl) i mi de i oni c I i qui d

\begin{tabular}{|c|c|}
\hline 著者 & $\begin{array}{l}\text { Asano Aki ra, Yang Ji nf eng, Kondo Takaf umi } \\
\text { Nor i zawa Ki mi hi ro, Nagai shi Ryuj i, Takahashi } \\
\text { Kenj i , Yoshi da Yoi chi }\end{array}$ \\
\hline $\begin{array}{l}\text { j our nal or } \\
\text { publ i cat i on title }\end{array}$ & Radi at i on Physi cs and Chemi stry \\
\hline vol une & 77 \\
\hline number & $10-12$ \\
\hline page $r$ ange & 12441247 \\
\hline year & $2008-10-01$ \\
\hline URL & ht t p: //hdl . handl e. net /2297/11779 \\
\hline
\end{tabular}




\title{
Molar absorption coefficient and radiolytic yield of solvated electrons in diethylmethyl(2-methoxy)ammonium bis (trifluoromethanesulfonyl)imide ionic liquid
}

\author{
Akira Asano $^{1)}$, Jinfeng Yang ${ }^{1 *}$, Takafumi Kondoh ${ }^{1)}$, Kimihiro Norizawa ${ }^{1)}$, Ryuji \\ Nagaishi $^{2)}$, Kenji Takahashi ${ }^{3)}$, Yoichi Yoshida ${ }^{1)}$ \\ ${ }^{1)}$ The Institute of Scientific and Industrial Research (ISIR), Osaka University, 8-1 Mihogaoka Ibaraki \\ 567-0047, Japan \\ 2) Japan Atomic Energy Agency (JAEA), Tokai-mura, Ibraki 319-1195, Japan \\ ${ }^{3)}$ Graduate School of Natural Science and Technology, Kanazawa University, Kakuma-machi, \\ Kanazawa 920-1192, Japan
}

\begin{abstract}
The molar absorption coefficient and the radiolytic yield of solvated electron in ionic liquid of DEMMA-TFSI were estimated respectively to be $2.3 \times 10^{4} \mathrm{~L} \mathrm{~mol}^{-1} \mathrm{~cm}^{-1}$ and $0.8 \times 10^{-7} \mathrm{~mol} \mathrm{~J}^{-1}$ by observing the reaction and kinetics of solvated electron and dry electron in the ionic liquid. The solvated electron in DEMMA-TFSI has a very broad absorption spectrum peaking at $1100 \mathrm{~nm}$ and reacts slowly with pyrene with a rate constant of $3.5 \times 10^{8} \mathrm{~L} \mathrm{~mol}^{-1} \mathrm{~s}^{-1}$, while the dry electron is captured rapidly by pyrene with $C_{37}$ value of $0.065 \mathrm{~mol} \mathrm{~L}^{-1}$.
\end{abstract}

Keywords: Ionic liquid, Solvated electron, Dry electron, Molar absorption coefficient, Sadiolytic yield, Pulse radiolysis

\footnotetext{
*Corresponding author.

E-mail address: yang@sanken.osaka-u.ac.jp (Jinfeng Yang)
} 


\section{Introduction}

Room temperature ionic liquids, novel green recyclable, have gained a great deal of attention as a new class of compounds for replacement of conventional organic solvents due to their unique properties, i.e. negligible volatility, thermal and chemical stability, and so on. Therefore, the ionic liquids have been proposed for applications in various fields such as organic chemical reactions, catalysis, extractions and electrochemistry (Welton, 1999; Wasserscheid and Keim, 2000; Sheldon, 2001; Funston et al., 2007). Recently, the ionic liquids have aroused much interest as new media in nuclear fuel reprocessing (Shkrob et al., 2007). The ionic liquid is also an interesting solvent for studying radiation chemistry, such as reaction and kinetics of solvated electrons and dry electrons, electron transfer, and so on, because of the existence of free cation and anion at room temperature.

Several pulse radiolysis studies on ionic liquids (Behar et al., 2001; Behar et al., 2002; Grodkowski and Neta, 2002a-c; Wishart and Neta, 2003; Grodkowski et al., 2003; Wishart et al., 2005; Kato, et al., 2007; Takahashi et al., 2007; Kimura et al., 2007) have been reported. The researches indicate that, for the imidazolium ionic liquids, dry electrons produced by ionization before full solvation are rapidly scavenged by the solvent molecules. In the ammonium ionic liquids, the electrons are solvated quickly to form solvated electrons. The solvated electrons in the ammonium ionic liquids have a very broad absorption band peaking over $1000 \mathrm{~nm}$. In $\mathrm{R}_{4} \mathrm{NNTf}_{2}$ (Wishart and Neta, 2003), a radiolytic yield of the solvated electrons was observed to be $0.7 \times 10^{-7}$ mol $\mathrm{J}^{-1}$ in the nanosecond time region and the peak molar absorption coefficient of the solvated electron was reported to be $2.2 \times 10^{4} \mathrm{~L} \mathrm{~mol}^{-1} \mathrm{~cm}^{-1}$ at $1410 \mathrm{~nm}$. The reactions of the solvated electrons with some aromatic scavengers such as benzophenone and pyrene are diffusion-limited, with rate constants in order of $10^{8} \mathrm{~L} \mathrm{~mol}^{-1} \mathrm{~s}^{-1}$. Moreover, the radiolysis studies indicate that the dry electron capture with scavengers is very efficient in ionic liquids. However, the study on the primary processes or reactions in ionic liquids (e.g. reaction of solvated electron and dry electron) and the estimation of the initial yields of! the solvated electrons or species are essential in order to design the ionic liquids for new applications.

We have, therefore, proposed femto-, pico- and nanosecond pulse radiolysis to observe the dynamics of solvated electron and dry electron in ionic liquids. The present work reports the spectrum, the molar absorption coefficient, and the!radiolytic yield of solvated electrons in ionic liquid of N,N-Diethyl-n-methyl-n-(2-methoxy)ammonium bis (trifluoromethanesulfonyl)imide (DEMMA- TFSI) based on the nanosecond pulse radiolysis studies. The reaction and kinetics of solvated electron and dry electron with pyrene! solute in DEMMA-TFSI were observed.

\section{Experimental}

In the present work, a hydrophobic ionic liquid of N,N-Diethyl-n-methyl-n-(2-methoxy) ammonium bis(trifluoromethanesulfonyl)imide (DEMMA-TFSI; produced by Kanto Chemical Co., 
the viscosity of $120 \mathrm{mPa}$ s at $20^{\circ} \mathrm{C}$ ) was used. Before the experiment, the ionic liquid was purified by repeated extractions with water (4 times) to remove $\mathrm{LiCl}$ and excess reagent, and then dried three hours under vacuum at $80^{\circ} \mathrm{C}$.

The optical absorption spectrum and transient absorption time profile were measured by Osaka University nanosecond pulse facility based on an $8 \mathrm{~ns}$ electron pulse from a $28 \mathrm{MeV}$ L-band linear accelerator. Analyzing light was provided by a pulsed xenon flash lamp. A monochrometer with narrow $(10 \mathrm{~nm})$ band-pass filters was used to select the analyzing light wavelength. The transient absorption signals were measured by a silicon photodiode at wavelength of $\leq 1000 \mathrm{~nm}$ and an InGaAs photodiode at $\geq 1000 \mathrm{~nm}$. All measurements were carried out at room temperature. A 10-mm-thick sample cell and a radiolytic dose of around 21 Gy were used in the transient absorption measurement.

\section{Results and Discussion}

\subsection{Spectra of solvated electrons in DEMMA-TFSI}

Figure 1 shows the optical absorption spectra of solvated electrons obtained in the nanosecond pulse radiolysis of DEMMA-TFSI with 0 and $16 \mathrm{mmol} \mathrm{L}^{-1}$ pyrene. The solvated electron in DEMMA-TFSI has a very broad absorption band peaking at $1100 \mathrm{~nm}$, i.e. $1.1 \mathrm{eV}$ with energy band width of $0.6 \mathrm{eV}$ in full-width at half-maximum (FWHM). The electrons in DEMMA-TFSI can be considered to be weekly solvated, similar to ionic liquids of TMPA-TFSI (Katoh et al., 2007), P13-TFSI and P14-TFSI (Takahashi et al., 2007), but is stronger than ionic liquid of $\mathrm{R}_{4} \mathrm{NNTf}_{2}(1410$ $\mathrm{nm}$ ) (Wishart and Neta, 2003). By adding the pyrene solute, the solvated electrons react with pyrene to produce pyrene anion radicals, resulting in the decrease of the optical density at $1100 \mathrm{~nm}$ and the increase at around $480 \mathrm{~nm}$. The absorption band around $1100 \mathrm{~nm}$ in Fig. 1 is, therefore, assigned to the solvated electrons. The small absorption band that peaks at $440 \mathrm{~nm}$ is assigned to the pyrene cation radical produced by the reaction of the solvent cation radical with pyrene.

\subsection{Yield and reactions of solvated electrons}

Figures 2 and 3 show respectively the solvated electron decay in DEMMA-TFSI at $1100 \mathrm{~nm}$ and the formation of pyrene anion radical at $480 \mathrm{~nm}$ as functions of the pyrene concentration. In the case of the absence of solute, the solvated electron decays via as a simple first-order process with a rate constant of $k_{1}=6.4 \varnothing 10^{6} \mathrm{~s}^{-1}$ (lifetime: $160 \mathrm{~ns}$ ). The relative short lifetime suggests that the solvated electrons react with some solvent molecules in ionic liquid. However, it must be reacting with impurities or the residual water rather than through a second-order process with a hole species.

The reaction of the solvated electrons with pyrene solute is dependent on the concentration of pyrene,

$$
e_{\text {sol }}^{-} \stackrel{k_{1}}{\longrightarrow}
$$




$$
e_{\text {sol }}^{-}+P y \stackrel{k_{2}}{\longrightarrow} P y^{\bullet-} \stackrel{k_{3}}{\longrightarrow},
$$

where $e_{\text {sol }}^{-}, P y$, and $P y^{-}$represent the solvated electron, the pyrene, and the pyrene anion radical, respectively. The decay rate of the solvated electron in the ionic liquid increases with the pyrene concentration, while the yield of pyrene anion radical formed increases with the pyrene concentration. The decrease of the initial solvated electron yield in Fig. 2(a) and the increase of the initial pyrene anion radical yield in Fig. 3 are due to the reaction of the dry electron with pyrene, as described below. The first-order rate constants calculated from the solvated electron decay show a linear correlation with the pyrene concentration, as given in Fig. 2(b). The second-order rate constant of the solvated electron reaction with pyrene is obtained to be $k_{2}=3.5 \varnothing 10^{8} \mathrm{~L} \mathrm{~mol}^{-1} \mathrm{~s}^{-1}$. The observed rate constant is lower than that in water and alcohols by 2 orders of magnitude. According to the diffusion theory because of the viscosity of DEMME-TFSI being over 2 orders of magnitude higher than that of water and alcohols, the reaction of the solvated electrons with pyrene is also diffusion limited. However, the diffusion-controlled limit for DEMMA-TFSI can be estimated for the viscosity and the equation $K_{\text {diff }}=8000 R T / 3 \eta$ to be about $5.5 \times 10^{7} \mathrm{~L} \mathrm{~mol}^{-1} \mathrm{~s}^{-1}$. The observed rate constant for the reaction of the solvated electron with pyrene thus exceeds the limit by near one order of magnitude. Similar researches were also reported in several butylmethylimidazolium ionic liquids (McLean et al., 2002; Skryzpczak et al., 2003), $\mathrm{R}_{4} \mathrm{NNTf}_{2}$ ionic liquid (Wishart and Neta, 2003), and TMPA-TFSI ionic liquid (Katoh et al., 2007).

Now, we can estimate the! radiolytic yield and the molar absorption coefficient $\left(\varepsilon_{\text {sol }}\right)$ of solvated electrons in ionic liquid from the rate constants of the decay and reaction of the solvated electrons, as described above, with the obtained rate constants of $\mathrm{k}_{1}$ and $\mathrm{k}_{2}$ as,

$$
\begin{aligned}
G_{\text {sol }}(t)= & G_{\text {sol }}(0) \exp \left\{-c / C_{37}-\left(k_{1}+k_{2} c\right) t\right\}, \\
G_{P y}(t)= & G_{\text {sol }}(0) \exp \left(-c / C_{37}\right) k_{2} c /\left(k_{1}+k_{2} c-k_{3}\right)\left[\exp \left(-k_{3} t\right)-\exp \left\{-\left(k_{1}+k_{2} c\right) t\right\}\right] \\
& +G_{\text {sol }}(0)\left\{1-\exp \left(c / C_{37}\right)\right\} \exp \left(-k_{3} t\right),
\end{aligned}
$$

where $G_{\text {sol }}(t)$ and $G_{P y}(t)$ are the yields of the solvated electron and the pyrene anion radical at time $t$, $G_{s o l}(0)$ and $G_{P y}(0)$ are the initial yields of the solvated electron and the pyrene anion radical at time zero, $\mathrm{c}$ is the concentration of pyrene in ionic liquid, $C_{37}=0.065 \mathrm{~mol} \mathrm{~L}^{-1}$ is the fractional yield of the solvated electrons remaining after the reaction of dry electrons, as described below. Therefore, we can fit the kinetics of the pyrene anion radicals (i.e. the formation and decay of the pyrene anion radicals as given in Fig. 3 at $480 \mathrm{~nm}$ ) as a function given in Eq. (4). The fitting results are given! as the solid lines in Fig. 3, where the rate constant of pyrene anion radical is! estimated to be $k_{3}=2 \sim 3$ $\emptyset 10^{6} \mathrm{~s}^{-1}$. By using the molar absorption coefficient of $5 \times 10^{4} \mathrm{~L} \mathrm{~mol}^{-1} \mathrm{~cm}^{-1}$ for pyrene anion radical (Wishart and Neta, 2003), the radiolytic yield of the solvated electrons in DEMME-TFSI was thus obtained to be $0.8 \times 10^{-7} \mathrm{~mol} \mathrm{~J}^{-1}$ at time zero, i.e. the pulse end of electron beam (the actual time $\sim 10$ ns). The peak molar absorption coefficient of solvated electrons in DEMMA-TFSI was obtained to 
$2.3 \times 10^{4} \mathrm{~L} \mathrm{~mol}^{-1} \mathrm{~cm}^{-1}$ from the measured optical density at $1100 \mathrm{~nm}$ (in Fig. 2(a)). It would be noted that the molar absorption coefficient of the solvated electron in ionic liquid of DEMMA-TFSI is slightly higher than that in the polar solvents such as water, but the yield is lower than water by a factor of 4 . The similar results in $\mathrm{R}_{4} \mathrm{NNTf}_{2}$ ionic liquid $\left(0.7 \times 10^{-7} \mathrm{~mol} \mathrm{~J}^{-1}\right.$ and $2.2 \times 10^{4} \mathrm{~L} \mathrm{~mol}^{-1} \mathrm{~cm}^{-1}$ at $1410 \mathrm{~nm}$ ) were reported by Wishart and Neta.

\section{3. Dry electron capture by pyrene.}

The dry electron before full solvation is captured efficiently by pyrene solute, resulting in the initial solvated electron decreases in Fig. 2(a) and the initial pyrene anion radical increases in Fig. 3. The experimental data indicates that, comparing with the reaction of the solvated electron with pyrene, the dry electron in DEMMA-TFSI reacts rapidly with the pyrene. The rate constant of the dry electron capture with solutes can not observed within the nanosecond time region. However, the fractional yield of the solvated electrons remaining after the dry electron capture can be evaluated by using the relation $G_{c} / G_{0}=\exp \left(-c / C_{37}\right)$, where $G_{c}$ is the yield of solvated electrons at a given solute concentration $c, G_{0}$ is the yield of solvated electrons without scavenger, and $C_{37}$ is the concentration at which only $37 \%(=1 / e)$ of the electrons survive to be solvated. Figure 4 shows plots of $\ln \left(G_{c} / G_{0}\right)$ as a function of the pyrene concentration in DEMMA-TFSI. The $C_{37}$ value of pyrene in DEMMA-TFSI is obtained to be $0.065 \mathrm{~mol} \mathrm{~L}^{-1}$. The obtained $C_{37}$ value is comparable with previous reported values: $0.062 \mathrm{~mol} \mathrm{~L}^{-1}$ for benzophenone, $0.063 \mathrm{~mol} \mathrm{~L}^{-1}$ for pyrene, $0.084 \mathrm{~mol} \mathrm{~L}^{-1}$ for phenanthrene in $\mathrm{R}_{4} \mathrm{NNTf}_{2}$ (Wishart et al., 2003), and $0.057 \mathrm{~mol} \mathrm{~L}^{-1}$ for Bmim cation in TMPA-TFSI (Katoh et al., 2007).

The dry electron capture by pyrene also provides a convenient method to estimate the molar absorption coefficient and the initial! radiolytic yield of the solvated electrons in DEMMA-TFSI by assuming that the dry electron only solvates to form the solvated electron or reacts with pyrene to produce pyrene anion radical,

$$
\begin{aligned}
& e^{-} d r y \rightarrow e^{-} \text {sol } \\
& e^{-} d r y+P y \rightarrow P y .
\end{aligned}
$$

The molar absorption coefficient of the solvated electron in DEMMA-TFSI at $1100 \mathrm{~nm}$ is thus obtained to be $\varepsilon_{\text {sol }}=\varepsilon_{P y-} \Delta O D_{P y} / \Delta O D_{\text {sol }}=2.3 \times 10^{4} \mathrm{~L} \mathrm{~mol}^{-1} \mathrm{~cm}^{-1}$, where $\triangle O D_{P y-}$ and $\triangle O D_{\text {sol }}$, calculated from the experimental data in Figs. 3 and 2(a), are respectively the increase of optical density at $480 \mathrm{~nm}$ at $10 \mathrm{~ns}$ ( the initial pyrene anion radical yield due to the dry electron reaction) and the decrease of the optical density at $1100 \mathrm{~nm}$ at $10 \mathrm{~ns}$ (decrease of the initial solvated electron yield due to the dry electron reaction with pyrene). The initial yield of the solvated electrons was estimated to be $0.8 \times 10^{-7} \mathrm{~mol} \mathrm{~J}^{-1}$ at $10 \mathrm{~ns}$. The molar absorption coefficient and the initial yield 
agree with that from the analysis of the reaction of the solvated electron with pyrene. It indicates that the pulse radiolysis is a useful tool to study the ultrafast reactions and kinetics in ionic liquids.

\section{Conclusion}

The molar absorption coefficient and the radiolytic yield of solvated electrons in diethylmethyl (2-methoxy)ammonium bis(trifluoromethanesulfonyl)imide (DEMMA-TFSI)DEMMA-TFSI ionic liquid were estimated by observing the reaction and kinetics of solvated electron and dry electron in ionic liquid with a nanosecond pulse radiolysis technique. It is found that the solvated electron in DEMMA-TFSI has a very broad absorption spectrum peaking at $1100 \mathrm{~nm}$. The electrons in ionic liquid DEMMA-TFSI is weekly solvated, similar to ionic liquids of TMPA-TFSI, P13-TFSI and P14-TFSI, but is stronger than ionic liquid of $\mathrm{R}_{4} \mathrm{NNTf}_{2}(1410 \mathrm{~nm})$. The peak molar absorption coefficient and radiolytic yield of the solvated electrons in DEMMA-TFSI were obtained to be $2.3 \times$ $10^{4} \mathrm{~L} \mathrm{~mol}^{-1} \mathrm{~cm}^{-1}$ and $0.8 \times 10^{-7} \mathrm{~mol} \mathrm{~J}^{-1}$, respectively.

The reaction of the solvated electron with solute (i.e. pyrene) is found to be diffusion-controlled, with a lower rate constant of $3.5 \times 10^{8} \mathrm{~L} \mathrm{~mol}^{-1} \mathrm{~s}^{-1}$, but the reaction of the dry electron with pyrene is very efficient with $C_{37}$ value of $0.065 \mathrm{~mol} \mathrm{~L}^{-1}$, which suggests that the electrons in DEMME-TFSI could diffuse before full salvation. It indicates that the ionic liquid is an interesting reaction medium for examining the reaction mechanism of dry electrons. However, pico- and femtosecond time-resolved measurements should be examined for detailed knowledge of the reactions of dry electron in ionic liquids. 


\section{References}

Behar, D., Gonzalez, C., Neta, P., 2001. Reaction kinetics in ionic liquids: pulse radiolysis studies of 1-butyl-3-methylimidazolium salts. J. Phys. Chem. A 105, 7607-7614.

Behar, D., Neta, P., Schultheisz, C., 2002. Reaction kinetics in ionic liquids as studied by pulse radiolysis: redox reactions in the solvents methyltributylammonium bis(trifluoromethylsulfonyl) imide and N-butylpyridinium tetrafluoroborate. J. Phys. Chem. A 106, 3139-3147.

Funston, A. M., Fadeeva, T. A., Wishart, J. F., Castner, E. W. Jr., 2007. Fluorescence probing of temperature-dependent dynamics and friction in ionic liquid local environments. J. Phys. Chem. B 111, 4963-4977.

Grodkowski, J., Neta, P., 2002a. Reaction kinetics in the ionic liquid methyltributylammonium bis(trifluoromethylsulfonyl) imide. Pulse radiolysis study of dCF3 radical reactions. J. Phys. Chem. A 106, 5468-5473.

Grodkowski, J., Neta, P., 2002b. Reaction kinetics in the ionic liquid methyltributylammonium bis(trifluoromethylsulfonyl) imide. Pulse radiolysis study of 4-mercaptobenzoic acid. J. Phys. Chem. A 106, 9030-9035.

Grodkowski, J., Neta, P., 2002c. Formation and reaction of $\mathrm{Br} 2$ radicals in the ionic liquid methyltributylammonium bis(trifluoromethylsulfonyl)imide and in other solvents. J. Phys. Chem. A 106, 11130-11134.

Grodkowski, J., Neta, P., Wishart, J. F., 2003. Pulse radiolysis study of the reactions of hydrogen atoms in the ionic liquid methyltributylammonium bis[(trifluoromethyl)sulfonyl]imide. J. Phys. Chem. A 107, 9794-9799.

Katoh, R., Yoshida, Y., Katsumura, Y., Takahashi, Kenji., 2007. Electron photodetachment from iodide in ionic liquids through charge-transfer-to-solvent band excitation. J. Phys. Chem. B 111, 4770-4774.

Kimura, A., Taguchi, M., Kondoh, T., Yang, J., Yoshida, Y., Hirota, K., 2007. Study on the reaction of chlorophenols in room temperature ionic liquids by gamma and electron pulse radiolysis. submitted to Radiat. Phys. Chem.

McLean, A. J.; Muldoon, M. J.; Gordon, C. M.; Dunkin, I. R., 2002. Chem.Commun.1880-1881.

Mezyk, S. P., Jones. J., Cooper W. T., Tobien. T., Nickelsen. M. G., Adams. J. W., O'Shea. K. E., Bartels D. M., Wishart. J. M., Tornatore. P. M., Newman. K. S., Gregoire. K., and Weidman. D. J., 2005. Radiation chemistry of methyl-tert-butyl ether (MTBE) in aqueous solution. J. Chem. Phys. 122, 184512.

Sheldon, R., 2001. Catalytic reactions in ionic liquids. Chem. Commun. 2399-2407.

Shkrob, I. A., Chemerisov, S. D., Wishart, J. F., 2007. The initial stages of radiation damage in ionic liquids and ionic liquid-based extraction systems. J. Phys. Chem. B 111, 6878-6886

Skrzypczak, A., Neta, P., 2003. Diffusion-controlled electron transfer reactions in ionic liquids. J. 
Phys. Chem. A 107, 7800-7803.

Takahashi, K., Hiejima, Y., Katoh, R., Yang, J., Kondoh, T., Yoshida, Y., Katsumura, Y., 2007. Reactions of solvated electrons with imidazolium cations in ionic liquids. submitted to Radiat. Phys. Chem.

Wasserscheid, P., Keim, W., 2000. Ionic liquids-New “solutions”' for transition metal catalysis. Angew. Chem. Int. Ed. 39, 3773-3789.

Welton, T., 1999. Room-temperature ionic liquids. Solvents for synthesis and catalysis. Chem. Rev. 99, 2071-2083.

Wishart, J. F., Lall-Ramnarine, S. I., Raju, R., Scumpia, A., Bellevue, S., Ragbir, R., Engel, R., 2005.

Effects of functional group substitution on electron spectra and salvation dynamics in a family of ionic liquids. Radiat. Phys. Chem. 72, 99-104.

Wishart, J. F., Neta, P., 2003. Spectrum and reactivity of the solvated electron in the ionic liquid methyltributylammonium bis(trifluoromethylsulfonyl)imide. J. Phys. Chem. B 107, 7261-7267. 


\section{Figure captions}

Fig.1. Transient absorption spectra observed in the nanosecond pulse radiolysis for neat DEMMA-TFSI and that with $16 \mathrm{mM}$ pyrene at time zero, i.e. the pulse end of electron beam (the actual time $\sim 10 \mathrm{~ns}$ ).

Fig.2. (a) Transient absorption kinetics of solvated electrons at $1100 \mathrm{~nm}$ with (top to bottom) 0, 4, 8, 12, and $16 \mathrm{mmol} \mathrm{L}^{-1}$ pyrene in DEMMA-TFSI. Radiolytic dose: $21 \mathrm{~Gy}$, and (b) Plots of observed rate constants for solvated electron decay versus pyrene concentration.

Fig. 3 Transient absorption kinetics (dots) of pyrene anion radicals at $480 \mathrm{~nm}$ with (bottom to top) 0 , 4, 8, 12, and $16 \mathrm{mmol} \mathrm{L}^{-1}$ pyrene in DEMMA-TFSI, radiolytic dose: $21 \mathrm{~Gy}$, and the fitting results! (solid line) by Eq. (4).

Fig. 4. Plots of $\ln \left(G_{d} / G_{0}\right)$ as a function of the pyrene concentration in DEMMA-TFSI. 


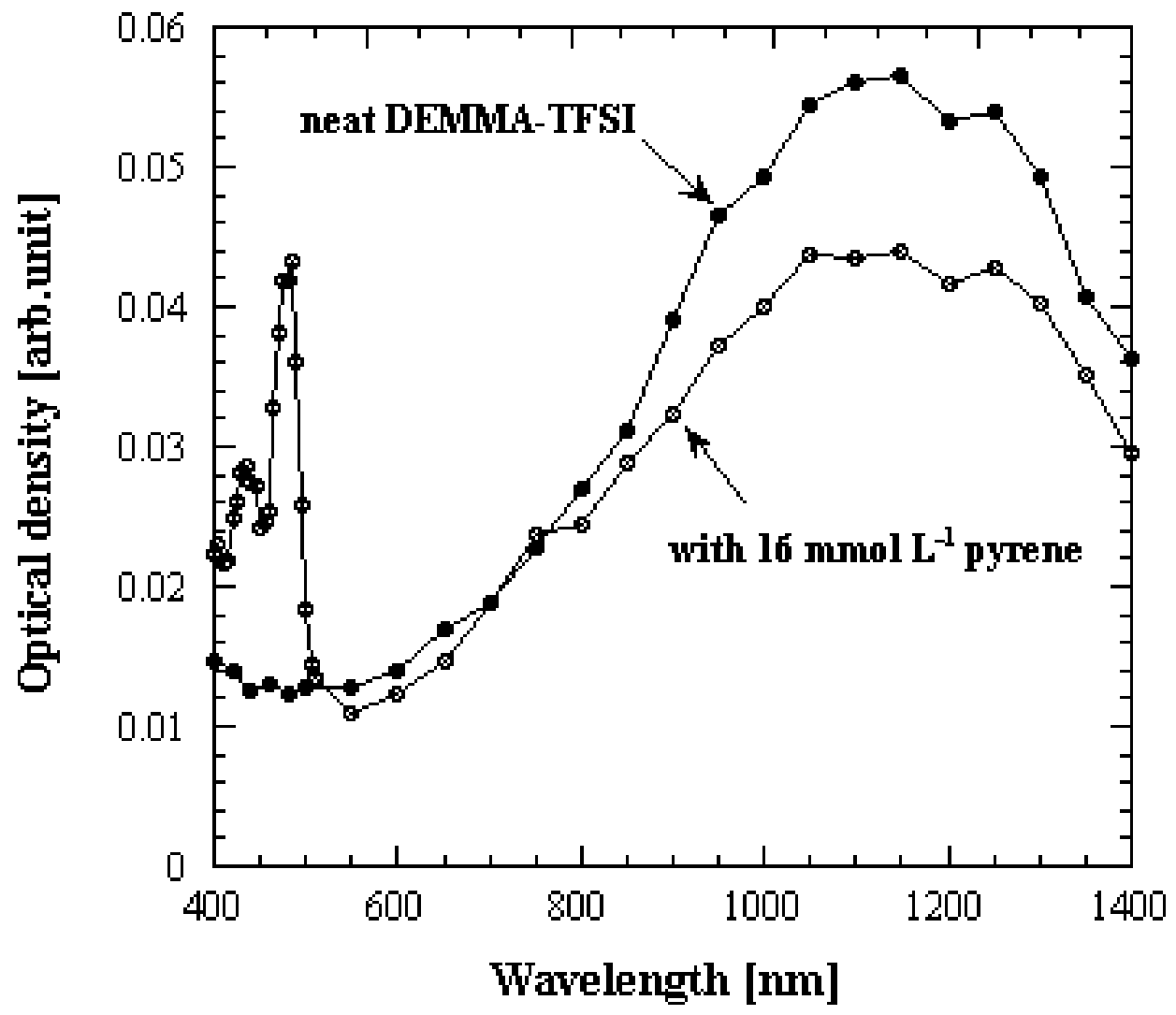

Fig. 1 


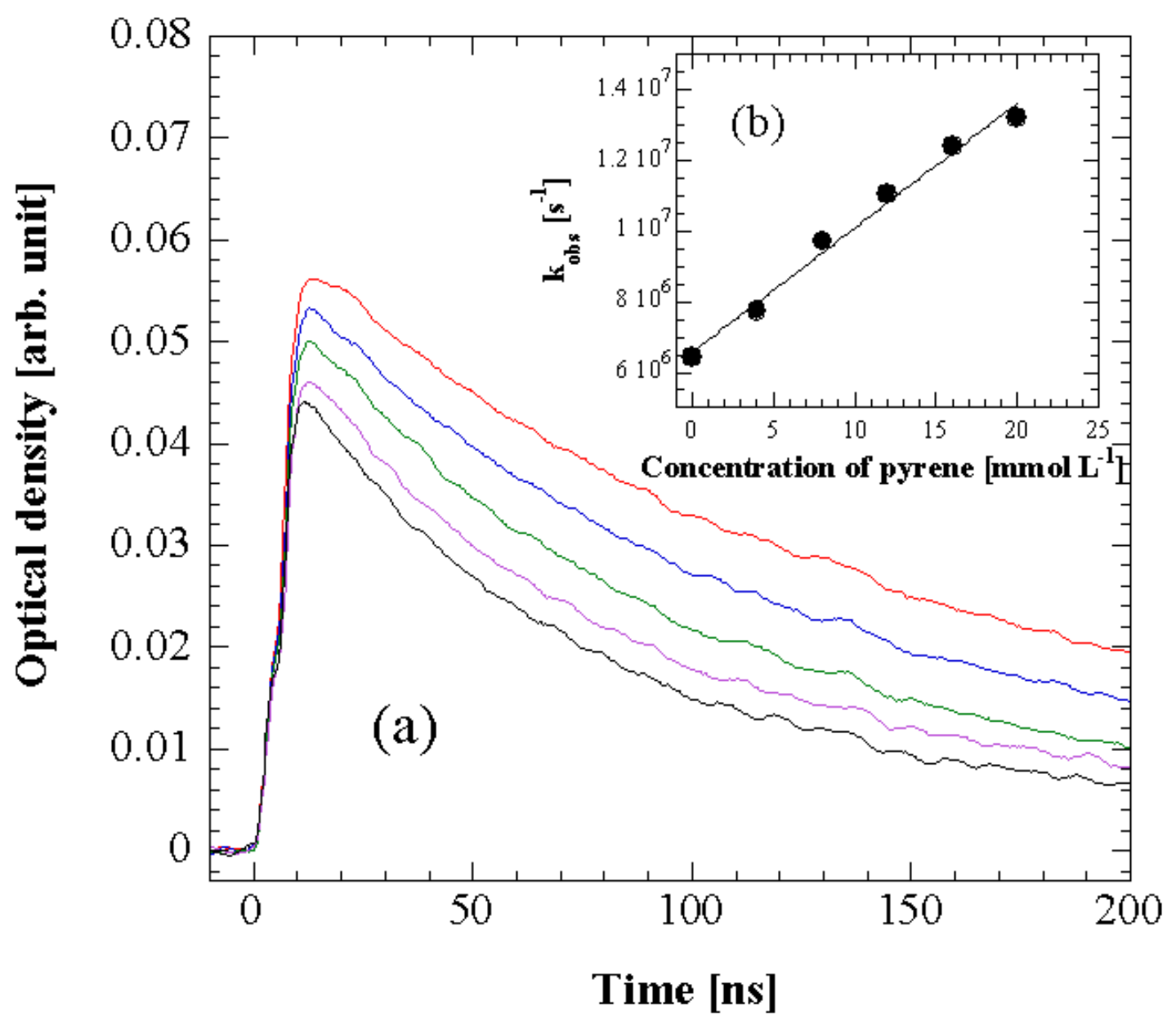

Fig. 2 


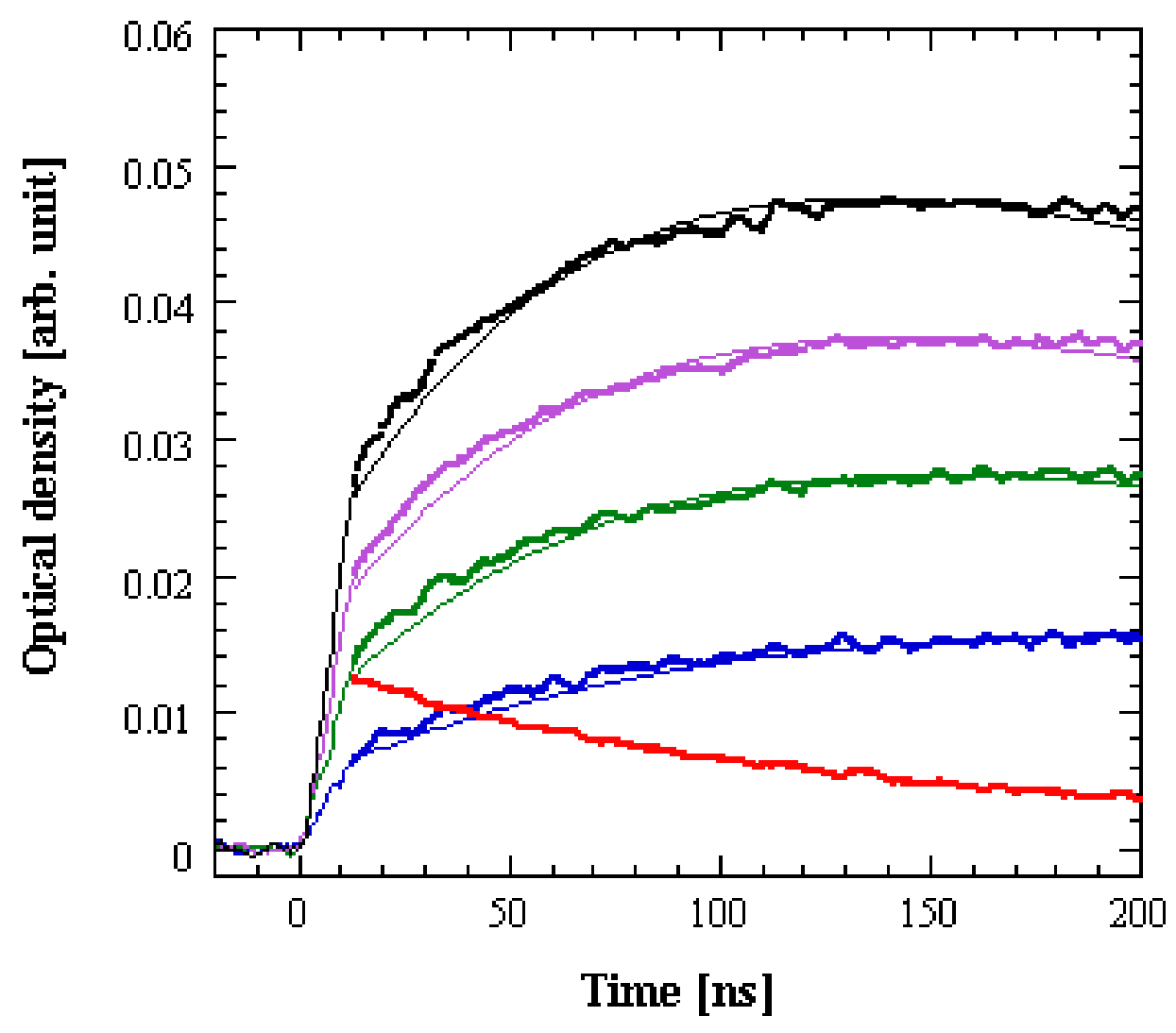

Fig. 3 


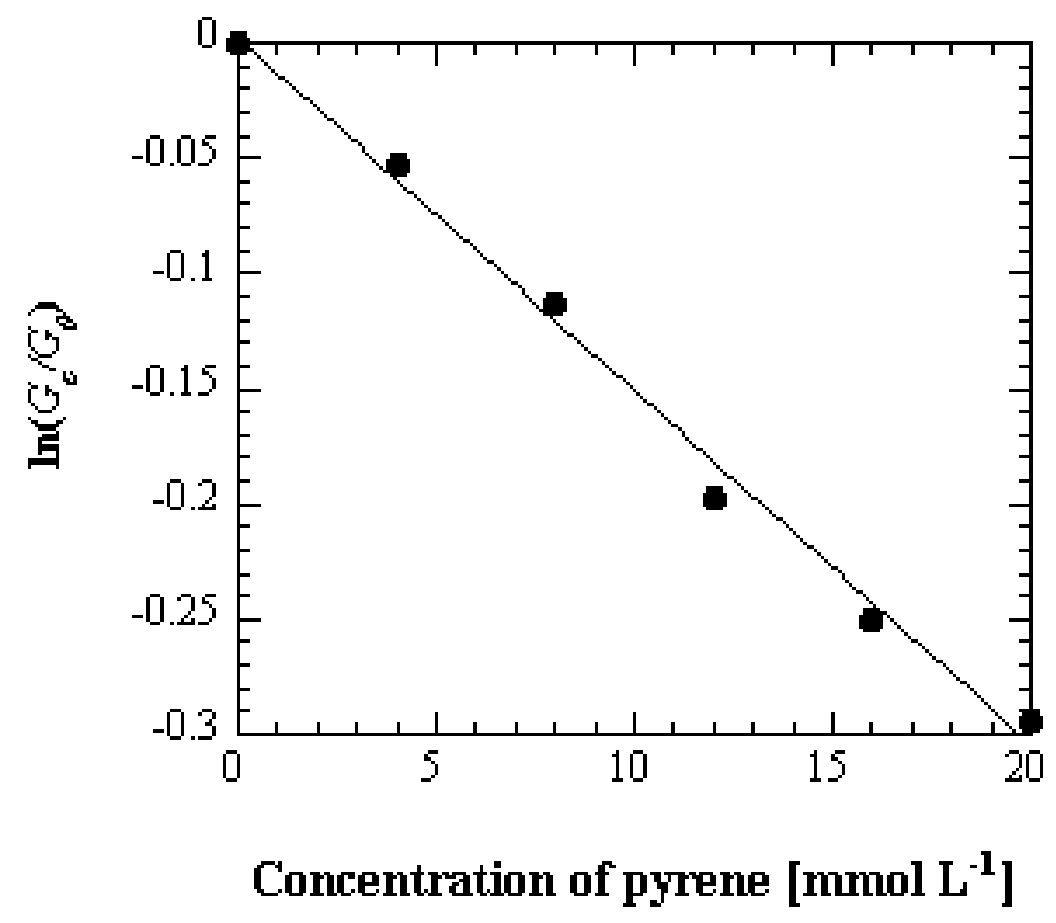

Fig. 4 